\title{
HUMAN SAFETY CRITERIA FOR RISK-BASED STRUCTURAL DESIGN
}

\author{
MIROSLAV SYKORA ${ }^{1}$, MILAN HOLICKY ${ }^{1}$, KAREL JUNG $^{1} \&$ DIMITRIS DIAMANTIDIS ${ }^{2}$ \\ ${ }^{1}$ Czech Technical University in Prague, Klokner Institute, Prague, Czech Republic. \\ ${ }^{2}$ OTH Regensburg, Faculty of Civil Engineering, Regensburg, Germany.
}

\begin{abstract}
Risk and reliability criteria are well established in many industrial sectors such as the offshore, chemical or nuclear industries. Comparative risk thresholds have been specified to allow a responsible organization or regulator to identify activities, which impose an acceptable level of risk concerning the participating individuals, or society as a whole. The scope of this contribution is to present target reliability criteria based on acceptable human safety levels. Application of theoretical principles is illustrated by examples of railway engineering structures. Initially it is shown how civil engineering structures for which human safety criteria play a role are classified according to Eurocodes. Examples include bridges, tunnels or station buildings. The general concepts for risk acceptance are then briefly reviewed, particularly in their relation to the target reliability criteria. The distinction between the two types of criteria is made: group risk and the acceptance criterion based on the Life Quality Index LQI approach introduced by ISO 2394:2015. The differences between the criteria for new and existing structures are discussed. The application is illustrated by an example of a bridge crossing an important railway line. It appears that while benefits and costs of a private stakeholder or public authority are reflected by economic optimisation, the society should define the limits for human safety to achieve uniform risks for various daily-life activities and across different industrial sectors.

Keywords: group risk, human safety, individual risk, Life Quality Index, railway, risk acceptance, structure, target reliability
\end{abstract}

\section{INTRODUCTION}

Risk and reliability acceptance criteria are well established in many industrial sectors such as the offshore, chemical or nuclear industries. Comparative risk thresholds have thereby been specified to allow a responsible organization or regulator to identify activities which impose an acceptable level of risk on the participating individuals, or on the society as a whole.

The scope of this contribution is to present target reliability criteria for railway engineering structures based on acceptable human risks. The structures under consideration are initially classified. Civil engineering structures such as bridges, station buildings, tunnels, etc. are taken into account, while electrical and mechanical components such as signalling systems, ventilation systems, electrification systems are not considered herein. The general concepts for risk acceptance are then briefly reviewed, particularly in their relation to the target reliability criteria. The application of theoretical principles is demonstrated by the example of a bridge crossing an important railway line. The difference between new and existing structures is highlighted and recommendations for practical applications are drawn.

\section{CLASSIFICATION OF STRUCTURES}

Railway civil engineering structures considered herein cover a wide range of constructions and can be divided into:

1. Track carrying structures such as bridges, viaducts, trestles or earth structures.

2. Ancillary structures such as tunnels, stations, towers, platforms or loading docks. 
The aforementioned structures can be classified according to the design working life - for example, 50 years for station buildings and 100 years for bridges and tunnels. They can be also classified according to the possible consequences in case of failure, i.e. minor, medium, large consequences. The second type of classification is for the determination of the target reliability of importance: structures with large potential consequences are associated with higher target reliability.

A classification of civil engineering structures according to various normative documents was provided by Sykora et al. [1]. As an example, UIC 7772 [2] makes distinction between:

1. Superstructures supporting elevated structures that are permanently occupied (offices, lodgings, business premises), structures for short-term gathering of people (theatres, cinemas) and multi-storey structures subject to short-term occupancy (multi-storey car parks, warehouses)

2. Superstructures not supporting elevated structures (roadways, road bridges, railway bridges, footbridges and similar structures) and single-storey structures not providing long-term occupancy (parking areas, warehouses).

In this contribution, the failure consequence classes according to EN 1990 [3] for basis of structural design are adopted:

- Low consequence for loss of human life, and economic, social or environmental consequences small or negligible; examples based on authors' experience: low rise buildings where only few people are present or small supply structures.

- Medium consequence for loss of human life, economic, social or environmental consequences considerable; examples: minor bridges.

- High consequence for loss of human life, or economic, social or environmental consequences very great; examples: normal and major bridges, and tunnels, major railway stations.

\section{RISK ACCEPTANCE CRITERIA}

Explicit risk acceptance criteria are commonly applied in many industrial sectors in order to provide either a quantitative decision tool for the regulator, or a comparable requirement for the industry when dealing with the certification/approval of a particular structure or system. The following criteria are commonly applied and analytically described $[4,5]$ :

1. Individual risk criteria: no individual (or group of individuals) involved in a particular activity can be exposed to an 'unacceptable' risk; a typical value is $10^{-6}$ per year [6]. If an individual worker or a member of the public is found to be exposed to excessive risk, safety measures are adopted regardless of the cost-benefit effectiveness.

2. Group risk criteria: a certain activity must not produce high frequency occurrences of large-scale accidents (i.e. with particularly severe consequences). This means that the "unacceptable" level of risk varies for different accident magnitudes. This principle attempts to capture a supposed socio-political aversion to large accidents and provides a regulatory basis (i.e. enforced investments in safety) in situations where the other criteria do not call for intervention.

The requirements based on criterion (1) can be significantly affected by the relative time fraction for which a person occupies or uses a structure. For railway civil engineering 
structures, it is assumed that this fraction is commonly low (exceptions may include most exposed workers), individual risk criteria become less important and group risk criteria dominate the derivation of target reliability values [7-9]. These criteria include human, economic and environmental criteria and are briefly reviewed in the following sections.

\section{GROUP RISK}

The group risk is often represented in the form of a numerical $F-N$-curve where $N$ represents the number of fatalities and $F$ is the frequency of accidents with more than $N$ fatalities $[4,5]$. This curve shows the probability of exceedance as a function of the number of fatalities $N$, commonly using a double logarithmic scale [10]:

$$
1-F_{N}(x)=\mathrm{P}(N>x)=\int_{x}^{\infty} \mathrm{f}_{N}(\xi) \mathrm{d} \xi
$$

where $\mathrm{f}_{N}(x)=$ probability density function of number of fatalities per year; and $\mathrm{F}_{N}(x)=$ cumulative density function, the value of which gives a probability of $\leq x$ fatalities per year. A simple measure for group risk is the annual expected value of the number of fatalities, which is frequently used to compare alternative projects in terms of their inherent risk with respect to human safety.

Typical $F-N$ curves reported in the literature show different patterns for the same industrial activity in various countries or for different industrial activities in the same country. The following general formula has been proposed to represent the group risk acceptance criterion:

$$
F \leq a N^{-k}
$$

where $a$ and $k=$ predefined constants that can be related to statistical observations from natural and man-made hazards [11]. Some natural hazards show relationships with $k$ slightly smaller than unity, while most manmade hazards are described by a relationship with $k>1$. From statistical observations the constants $a$ and $k$ vary widely depending on the type of hazard and the type of technical activity. It was proposed to set the constants in such a way that the curve envelops the curves for most natural hazards and some of the more common manmade hazards [7]. For acceptable risks related to structural failures, the constant would be around $a=10^{-6}$ and for marginally acceptable risks $a=10^{-4} ; k=1$ represents risk-neutral curves, $k>1$ describes curves with risk aversion and $k<1$ curves with risk proneness. The case of $k<1$ leads to infinitely large expected losses (in terms of lives or cost) and, therefore, is not acceptable.

The constant $a$ represents the frequency of occurrence of events with one and more fatalities; commonly, annual values are considered. Its value should be consistent with the reference system to which eqn (2) is applied. The reference system can range from a group of structures to an individual structural member and can include other structure-specific and industry-specific parameters [12]. For railway infrastructures, a national scale is assumed [5, 12, 13].

Based on the $F-N$ curves, the so-called ALARP - as low as reasonably possible - region can be defined by two limits [5]. The area above upper limit represents the risk that is not tolerated in any circumstances, while the risk below the lower limit is of no practical interest. Such acceptability curves have been developed in various industrial fields, including the chemical and the transportation industries.

In the ALARP principle the 'width' between the upper and lower bound curves is of importance. This width is often two orders of magnitudes allowing for excessive flexibility in 
practical cases. Examples of fatality criteria ( $F-N$ curves) given in Fig. 1 are based on the recommendations provided in:

- ISO 2394:1998 for general principles of structural reliability (superseded in 2015): $a=10^{-2}$ per annum and $k=2-$ examples provided as a risk acceptance criterion in structural design to avoid accidents where large numbers of people may be killed, deemed to be associated with a collapse of the whole structure

- Trbojevic [14]: $a=2 \times 10^{-5}$ per annum and $k=1-$ criterion for a structure with 100 persons at risk

- fib bulletin 80 [9]: $a=5 \times 10^{-4}$ per annum and $k=2-$ criterion derived for a main loadbearing member of a road bridge in order to maintain safety levels associated with current best practice

- ISO 2394:2015 [15], the LQI approach - see Section 5.

Note that the ISO and fib COM3 recommendations are based on the notion that $N$ represents the expected number of fatalities in an event and thus a limiting probability does not have the cumulative character of eqn (2). However, the numerical difference between these concepts is commonly negligible due to small probabilities under consideration. This is why the ISO and $f i b$ COM 3 curves are hereafter considered as directly comparable with $F-N$ curves. The ISO recommendation is applied in some countries; for instance Belgium and the Netherlands consider the acceptability criterion for the events with $N \geq 10$ fatalities [6].

The criteria in Fig. (1a) provide values ranging across more than two orders of magnitude. This is attributed to different reference systems for which these criteria are applicable. Whilst the example in ISO 2394:1998 is considered to be used for large groups of structures, the other two recommendations are focused on individual buildings [14], or on a key structural member of a bridge [9]. Hence, the reference system is of key importance when establishing the criteria for human safety.

Figure (1b) displays annual reliability indices $\beta$ derived from probability $F(N)$ given in Fig. (1a); see EN 1990 [3]. It appears that the criteria under consideration lead to a wide range of $\beta$-values particularly for events with $N<10$.
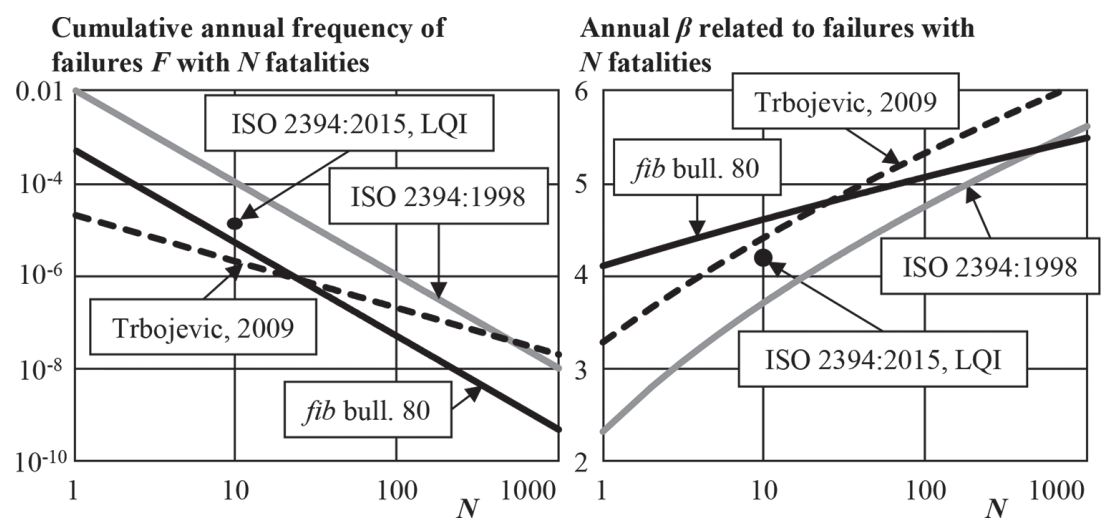

Figure 1: a) $F-N$ curves relating expected fatalities $(N)$ from an accidental event or failure and the annual frequency of occurrence $(F)$ of events with $\geq N$ fatalities; b) Annual reliability indices derived from the $F-N$ curves, related to events with $N$ fatalities. 
It is noted that human safety does not only involve fatalities but also injuries. In many studies injuries are related to fatalities by using a multiplicative factor, for example 0.1 for moderate injury and 0.5 for major injury. Based on this simple procedure weighted fatalities can be obtained. For detailed discussion see Ref. [16].

Diamantidis et al. [17] derived human risk acceptance criteria for the railway industry by assuming that the safety inherent in the traditional railways in the last two or three decades is acceptable. The safety target was therefore derived by analysing recent risk history of the railway system in terms of frequency of occurrence of accidents and the extent of their consequences. The procedure generally used to estimate the risk associated with railway transport is based on the analyses of the frequency of occurrence of given consequences for a given accident. The following classification of consequences was proposed:

- Medium consequences: 1 to 10 fatalities with an average value of 3 fatalities;

- Severe consequences: 11 to 100 fatalities with an average value of 30 fatalities;

- Catastrophic consequences: 101 to 1000 fatalities with an average value of 300 fatalities.

The evaluation of the probability can be performed by assuming that accidents occur according to a Poisson process [18]. In general a Bayesian approach by which expert judgements can be combined with statistical data is recommended, due to the limited experience with accidents. This approach was applied to data of recorded accidents of the Italian, Austrian and German railways [17]. The obtained results are considered valid for a first definition of an acceptable safety level for the Western European railway systems, and are comparable to computed values for various tunnel projects. The results and the recommended acceptance criteria can be summarized as follows:

- Events of medium consequences are associated an annual probability of $10^{-9}$ per trainkilometre;

- Events of severe consequences are associated an annual probability of $10^{-10}$ per trainkilometre;

- Events of catastrophic consequences are associated a probability of $10^{-11}$ per trainkilometre.

Example: Consider a railway tunnel with a length of 50 kilometres and a daily traffic flow of 200 trains in both directions. The acceptable return periods associated with accidental events are derived from the aforementioned results as follows:

- Number of train-kilometres per year: $50 \times 200 \times 365=3.65 \times 10^{6}$;

- Accidents associated with medium consequences: acceptable annual probability of an accident $p \leq 3.65 \times 10^{6} \times 10^{-9}=0.00365$; acceptable return period $\mathrm{T} \geq 1 / p \approx 300$ years;

- Severe consequences: 3000 years;

- Catastrophic consequences: 30000 years.

The derived acceptable return period for medium consequences is approximately within the range of the working life of important infrastructures, such as a long tunnel. For catastrophic consequences, the return period is of the same order of magnitude as is considered when designing industrial plants.

It is noted that, along with human risks, economic and environmental risks play an important role in decision making. Economic losses are direct consequences related, for example, 
to the repair of initial damage, replacement of structure and equipment, and indirect consequences such as loss of production, temporary relocation, rescue costs or loss of reputation. Environmental consequences can be presented in terms of permanent or long-term damage to terrestrial, freshwater, marine habitats and groundwater reservoirs. For more details, see Refs. [1, 19].

\section{LIFE QUALITY INDEX}

\subsection{ISO 2394 recommendations}

The Life Quality Index LQI, as for example discussed with respect to its implementation in technical standards in [20], was developed to support decisions related to allocations of available public resources between and within various societal sectors and industries. According to ISO 2394:2015 [15], the LQI is an indicator of the societal preference and capacity for investments into life safety expressed as a function of GDP, life expectancy at birth and ratio between leisure to working time.

The ISO standard [15] provides the detailed guidance on how preferences of the society regarding investments into health and life safety improvements can be described by the LQI concept. The target level is derived by considering the costs of safety measures, the monetary equivalent of societal willingness to save one life, and the expected number of fatalities in the event of structural failure. Essentially, this approach combines economic and human safety aspects. Compared with economic optimization [8, 21-23], it should lead to lower target reliability indices, as only the human consequences of structural failure are taken into account, while other losses such as economic and environmental costs are not taken into account. In the LQI approach, the danger to which the people are subjected might vary on an individual basis within the group of people affected, which may be deemed unethical [8].

The tentative minimum annual target reliabilities provided in ISO 2394 [15] are based on the following acceptance criterion:

$$
-\frac{\mathrm{d} p_{f}(d)}{\mathrm{d} d} \leq K_{l}=\frac{C_{1}(d) \times\left(\gamma_{\mathrm{s}}+\omega\right)}{S W T P \times N}
$$

where $p_{\mathrm{f}}=$ annual failure probability; $d=$ decision parameter (see the text below); the parameters indicated in Annex G of the ISO standard are adopted in the following analysis:

- $K_{1}=10^{-4.5}$ for small relative life-saving cost, deemed relevant for most design situations where higher reliability can be readily achieved;

- $\gamma_{\mathrm{s}}=0.03$ as an interest rate of relevance for decision-making on behalf of society, moderately selected rate of economic growth;

- $\omega=0.02$ as an annual rate of obsolescence that approximately corresponds here to the reciprocal value of a lifetime;

- Societal willingness to pay $S W T P=5100000 \mathrm{CHF}$;

- $N=10$ is the expected number of fatalities that could be associated with Consequence Class CC3 according to ISO 2394 [15] where CC3 is associated with $N<50$ and CC2 with $N<5$; five CCs are distinguished in the ISO standard. Medium failure consequences may be related to ten fatalities in [14]. Note that the empirical data in $[9,12]$ provide indications on the relationship between $N$ and collapsed floor area (buildings) or span length (bridges). 
For these notional values, marginal costs associated with a considered safety measure obtained from eqn (3) become $C_{1}(d)=32000 \mathrm{CHF}$ and ISO 2394 [15] indicates annual reliability index of 4.2 , which corresponds to the maximum acceptable failure rate $F(N=10)=$ $1.3 \times 10^{-5}$ per year (Fig. 1).

\subsection{Application to a railway bridge}

To provide a better insight into the performance of criterion (3), LQI reliability levels are derived for a key load-bearing member of a railway bridge following the simple example provided in Annex G of ISO 2394 [15]. A fundamental limit state function is considered:

$$
\mathrm{Z}(d)=R(d)-E
$$

where $R=$ resistance; $E=$ annual maxima of a load effect; and $d=$ decision parameter defined here as the ratio between the mean values of $R$ and $E, d=\mu_{R} / \mu_{E}$. The annual failure probability is obtained as $p_{\mathrm{f}}(d)=\mathrm{P}[\mathrm{Z}(d)<0]$.

The following probabilistic models of basic variables are taken into account:

- Lognormal distribution with coefficient of variation of 0.2 for resistance covering uncertainties in material properties, geometry and resistance model uncertainty related to flexural or shear resistance, axial compression, buckling etc.

- Gumbel distribution with coefficient of variation of 0.4 for annual maxima of a combined load effect covering:

- permanent actions

- time-invariant components of variable actions such as shape, exposure and other factors or load model simplifications

- annual maxima of time-variant components of variable actions, for instance snow load on the ground, basic wind velocity pressure, imposed or traffic loads.

The aforementioned notional values of the input parameters of eqn (3), deemed to describe the alternative of "small relative life-saving costs" according to the ISO standard, are adapted to the railway bridge example as follows:

- $\omega$ is decreased to 0.01 , corresponding to a standard lifetime of bridges of 100 years;

- $\gamma_{\mathrm{s}}$ is reduced to 0.0285 following the guidance of the Green Book of the UK Treasury for public sector projects.

while $S W T P$ and $C_{1}(d)$ are considered by the same values and $N$ is a study parameter.

Figure 2 shows annual reliability indices related to events with $N$ fatalities based on the LQI approach in ISO 2394 [15] and, for the sake of comparison, the criterion for human safety for bridges in $f i b$ bulletin 80 [9]. It is observed that for larger $N$, the fib bulletin 80 for existing structures requires higher reliability levels than the ISO standard for new structures ('small relative life-saving costs'). This is in contradiction with numerous previous studies advocating lower target levels for existing structures [8, 19, 23-25] and with the theoretically justified approach to reduce target reliability levels for increasing costs of safety measures. The discrepancy is primarily attributable to the risk-averse value $k=2$ adopted in the $f i b$ bulletin. When a risk-neutral unity is taken into account, the fib curve in Fig. 2 becomes parallel and close to the ISO level for medium relative life-saving costs. 


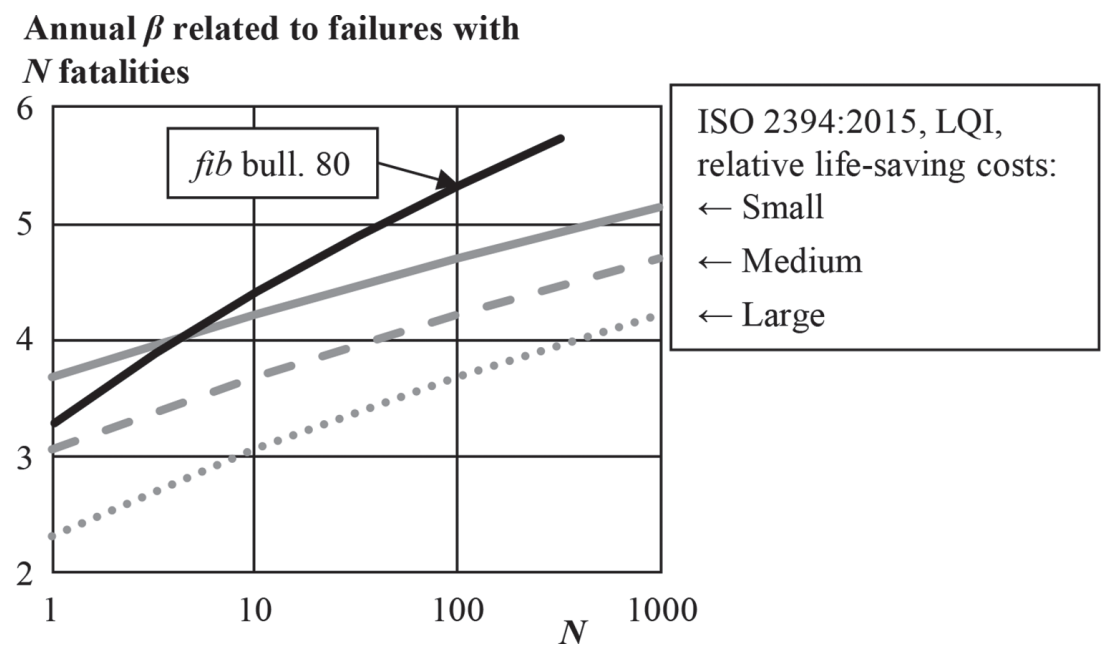

Figure 2: Annual reliability indices $\beta$ related to events with $\geq N$ fatalities, based on the LQI approach in ISO 2394 [15] and the criterion for human safety for existing bridges in fib bulletin 80 [9].

To indicate appropriate target reliability for existing structures, LQI $\beta$-values are further derived for 'medium' and 'large' relative life-saving costs. Following the example in Annex G of ISO 2394 [15], this can be achieved by increasing $C_{1}(d)$ by one or two orders of magnitude for medium and large relative life-saving costs, respectively [16]. This results in decreasing $\beta$-levels (Fig. 2):

- By about 0.5 for medium relative life-saving costs

- By more than 1.0 for large relative life-saving costs.

More information on the LQI approach including application in the bridge industry is provided in [26]. The differences in target reliability can be readily reflected by updating partial factors in practical verifications [27, 28].

\section{CASE STUDY}

Practical application of the human safety criteria is demonstrated by the example of a bridge pier located in the vicinity of a railway line (Fig. 3) and the related hazard of accidental impact of a train on the pier. A probabilistic risk analysis was employed to optimise the bridge design from the perspective of an owner of the bridge. The analysis described in Ref. [1] was based on an event tree approach, considering multiple hazards scenarios related to derailment of a train, impact to the bridge, collapse of the bridge due to impact and secondary collision with a train in the opposite direction. The UIC recommendations related to probabilities of unfavourable events [2] along with real traffic were taken into account. Figure 4 displays the computed $F-N$ curves for different train speeds. As an example for a train speed of $120 \mathrm{~km} / \mathrm{h}$, the curve was obtained as follows:

- With an annual probability of $1.3 \times 10^{-7}$ (equal to frequency due to its very low value), the most adverse scenario - derailment of the train and impact to the bridge, collapse of the 


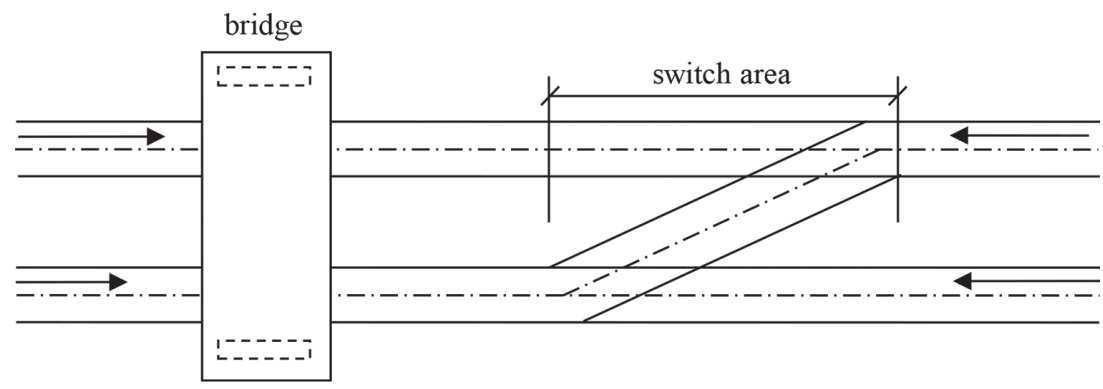

Figure 3: Plan view of the bridge.

\section{Cumulative annual frequency of failures $F$ with $N$ fatalities}

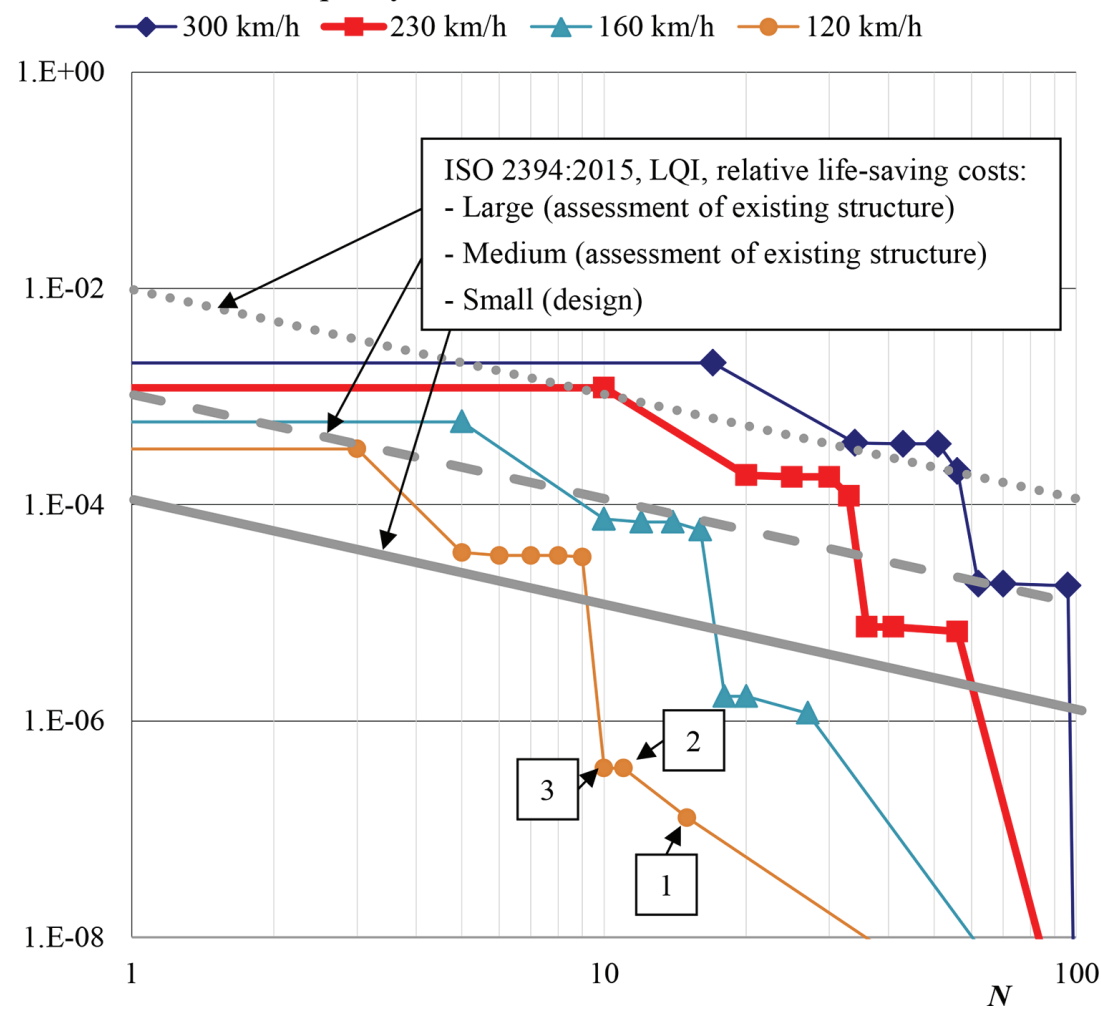

Figure 4: $F-N$ curves for different train speeds and the LQI acceptance criteria according to ISO 2394 [15].

bridge and secondary collision with a passenger train in the opposite direction - leads to 15 fatalities (point 1 in Fig. 4).

- With an annual probability of $2.4 \times 10^{-7}$, the second most adverse scenario - derailment, impact, secondary collision with a passenger train, but no bridge collapse - results in 11 fatalities. At least 11 fatalities are then associated with a cumulative frequency $F$ of $1.3 \times$ $10^{-7}+2.4 \times 10^{-7}=3.7 \times 10^{-7}$ (point 2 ).

- With negligible probability, the third most adverse scenario yields 10 fatalities and in Fig. 4 is thus associated with a cumulative frequency $F$ of $3.7 \times 10^{-7}$ (point 3). 
It remains to be verified whether the optimum design strategy complies with the criteria on human safety that may be postulated by public authorities. As an example the LQI acceptance criteria according to ISO 2394 [15] are plotted in Fig. 4. It is shown that for any train speed under consideration, the human safety criteria suggest that human risks exceed the commonly acceptable level for design situation and further design measures to mitigate human safety risks are required. Options include increasing the lateral distance of the bridge piers beyond the optimum value, or increasing the distance between the bridge and switch area (Fig. 3).

When an existing bridge is considered and a possibility of the change of the switch area is disregarded, increasing the lateral distance of the bridge piers from the railway tracks is an excessively costly measure. The acceptance criterion for large relative life-saving costs may then be applied and a train speed of $230 \mathrm{~km} / \mathrm{h}$ can be approved (Fig. 4).

\section{CONCLUSIONS}

The following conclusions can be drawn from the presented study:

- The human safety criteria should always be applied to check whether or not the design strategy based on economic optimisation including compensation costs related to fatalities, injuries and environmental impacts leads to acceptable risks to persons endangered by structural failure.

- While benefits and costs of a private stakeholder or public authority are reflected by economic optimisation, the society defines the limits for human safety through: (1) The individual risk criterion that provides comparison with risks related to other activities that are acceptable for the society. This criterion is particularly useful when risks vary across the range of persons potentially dependent on structural failure. However, the criterion is significantly affected by the relative time fraction for which a person occupies or uses a structure. As this fraction is very low for transportation structures, the criterion is commonly not decisive for bridges or tunnels. It can be used to assess risks of most exposed workers.

(2) The group risk criteria, often expressed by $F-N$ curves, lack a sound theoretical basis and do not consider costs of safety measures; they are however applied in many industrial sectors in which major accidents may occur. The major difficulty is to define a reference system for their application.

- The LQI criterion takes into the cost efficiency of changing design parameters and of the safety measures. However, this criterion is dependent on numerous uncertain input parameters and it may be difficult to provide general practical recommendations.

\section{ACKNOWLEDGEMENTS}

This work was supported by the Czech Science Foundation under Grants P105/12/G059 and 16-11378S, by the Regensburg Center of Energy and Resources (RCER) of OTH Regensburg and by the Technology- and Science Network Oberpfalz (TWO). Outcomes of COST Action TU1402 have been utilized.

\section{NOTE}

1 ORCID: http://orcid.org/0000-0001-9346-3204

\section{REFERENCES}

[1] Sykora, M., Holicky, M., Jung, K. \& Diamantidis, D., Railway RAM and safety handbook (Chapter 45: Target reliability for new and existing railway civil engineering structures, accepted for publication), CRC Press/ Balkema: Leiden, 2017. 
[2] UIC 777-2, UIC Code 777-2, Structures built over railway lines, Construction requirements in the track zone, International Union of Railways: 2002.

[3] EN 1990, Eurocode - Basis of structural design, CEN: Brussels, pp. 87, 2002.

[4] HSE, Reducing risks, protecting people, Health \& Safety Executive: Norwich (UK), pp. 88, 2001.

[5] CIB TG 32, Risk assessment and risk communication in civil engineering (Report 259), CIB: Rotterdam, 2001.

[6] DNV GL, Harmonised risk acceptance criteria for transport of dangerous goods, European Commission DG-MOVE: pp. 183, 2014.

[7] JCSS, Probabilistic assessment of existing structures. Joint Committee on Structural Safety, edited by D. Diamantidis, RILEM Publications S.A.R.L., 2001.

[8] Steenbergen, R.D.J.M., Sykora, M., Diamantidis, D., Holicky, M. \& Vrouwenvelder, A.C.W.M., Economic and human safety reliability levels for existing structures. Structural Concrete, 16, pp. 323-332, 2015. https://doi.org/10.1002/suco.201500022

[9] fib COM3 TG3.1, Partial factor methods for existing structures (fib bulletin 80, recommendation), fib: pp. 129, 2016.

[10] Jonkman, S.N., van Gelder, P.H.A.J.M. \& Vrijling, J.K., An overview of quantitative risk measures for loss of life and economic damage. Journal of Hazardous Materials, 99(1), pp. 1-30, 2003. https://doi.org/10.1016/s0304-3894(02)00283-2

[11] Vrouwenvelder, T., Leira, B.J. \& Sykora, M., Modelling of hazards. Structural Engineering International, 22(1), pp. 73-78, 2012. https://doi.org/10.2749/101686612x13216060213356

[12] Tanner, P. \& Hingorani, R., Acceptable risks to persons associated with building structures. Structural Concrete, 16(3), pp. 314-322, 2015. https://doi.org/10.1002/suco.201500012

[13] Vrijling, J., van Gelder, P. \& Ouwerkerk, S., Criteria for acceptable risk in the Netherlands. Infrastructure Risk Management Processes, American Society of Civil Engineers, pp. 143-157, 2005. https://doi.org/10.1061/9780784408155.ch05

[14] Trbojevic, V.M., Another look at risk and structural reliability criteria. Structural Safety, 31(3), pp. 245-250, 2009. https://doi.org/10.1016/j.strusafe.2008.06.019

[15] ISO 2394, General principles on reliability for structures. ISO: Geneve, Switzerland, pp. 111, 2015.

[16] Diamantidis, D., Holicky, M. \& Sykora, M., Target reliability levels based on societal, economic and environmental consequences of structural failure (accepted for publication). Proc. ICOSSAR 2017, CRC Press/Balkema: Leiden (The Netherlands), pp. 10, 2017.

[17] Diamantidis, D., Zuccarelli, F. \& Westhäuser, A., Safety of long railway tunnels. Reliability Engineering \& System Safety, 67(2), pp. 135-145, 2000. https://doi.org/10.1016/s0951-8320(99)00059-9

[18] Melchers, R.E., Structural reliability analysis and prediction, John Wiley \& Sons Ltd.: Chichester, England, pp. 437, 2001.

[19] Sykora, M., Holicky, M., Jung, K. \& Diamantidis, D., Target reliability for railway civil engineering structures (accepted for publication). Proceedings ESREL 2017, Taylor and Francis/ Balkema: Leiden, 2017. 
[20] Rackwitz, R., Optimization - the basis of code-making and reliability verification. Structural Safety, 22(1), pp. 27-60, 2000. https://doi.org/10.1016/s0167-4730(99)00037-5

[21] Holicky, M., Optimisation of the target reliability for temporary structures. Civil Engineering and Environmental Systems, 30(2), pp. 87-96, 2013. https://doi.org/10.1080/10286608.2012.733373

[22] Holicky, M., Diamantidis, D. \& Sykora, M., Determination of target safety for structures. Proceeding of ICASP12, eds. T. Haukaas, pp. 8, 2015.

[23] Sykora, M., Holicky, M., Jung, K. \& Diamantidis, D., Target reliability for existing structures considering economic and societal aspects. Structure and Infrastructure Engineering, 13(1), pp. 181-194, 2016. https://doi.org/10.1080/15732479.2016.1198394

[24] Steenbergen, R.D.J.M. \& Vrouwenvelder, A.C.W.M., Safety philosophy for existing structures and partial factors for traffic loads on bridges. Heron, 55(2), pp. 123-139, 2010.

[25] Holicky, M., Markova, J. \& Sykora, M., Target reliability levels in present standards. Transactions of the VSB - Technical University of Ostrava, Civil Engineering Series, 14(2), pp. 46-53, 2014.

[26] Fischer, K., Virguez, E., Sánchez-Silva, M. \& Faber, M.H., On the assessment of marginal life saving costs for risk acceptance criteria. Structural Safety, 44, pp. 37-46, 2013. https://doi.org/10.1016/j.strusafe.2013.05.001

[27] Sykora, M., Holicky, M. \& Markova, J., Verification of existing reinforced concrete bridges using the semi-probabilistic approach. Engineering Structures, 56, pp. 1419-1426, 2013. https://doi.org/10.1016/j.engstruct.2013.07.015

[28] Caspeele, R., Sykora, M., Allaix, D.L. \& Steenbergen, R., The design value method and adjusted partial factor approach for existing structures. Structural Engineering International, 23(4), pp. 386-393, 2013.

https://doi.org/10.2749/101686613x13627347100194 